\title{
Hamiltonian Structures for Homogeneous Spaces
}

\author{
R. ARENS \\ University of California, Los Angeles, California \\ Received August 25, 1970
}

\begin{abstract}
The definition and classification of classical relativistic particles requires the classification of certain invariant tensor fields on the inhomogeneous Lorentz group. The entire 10-parameter set is exhibited. At the same time, a much larger class of Lie groups is treated. The connection with particles will be presented in the succeeding article.
\end{abstract}

\section{Introduction}

The action of time in the phase space of a dynamical system can for Hamiltonian systems be carried out by "canonical" transformations.

In an Einstein-Lorentz invariant system, the entire space-time group (Poincaré group $\mathscr{P}$ ) acts in the phase space. Those systems where one has or can introduce a suitable Poisson bracket operation preserved by the entire group and not merely the time are of special interest. Here the cases in which the group acts transitively are of basic interest. This problem is what we study here.

The phase space in these cases has the form $G / \Gamma$ where $\Gamma$ is a closed subgroup of the "space-time" group $G$. Let $C^{2}(G ; \Gamma)$ be the closed invariant 2-forms on $G$ for which the Lie algebra of $\Gamma$ is singular, but which are non-singular on the residue linear space of the Lie algebra of $G$ modulo that of $\Gamma$. Then $C^{2}(G ; \Gamma)$ corresponds $1: 1$ to the possible Poisson brackets.

It is shown that for many pairs $G, \Gamma$ the "generating functions" (in particular the Hamiltonian, which "generates" time translation) are linear combinations of matrix elements from the adjoint representation of $G$. This includes the case of the Poincare group.

The application of these findings to the classification of elementary particles will be published separately. It requires taking into account which pairs of Poisson brackets are equivalent.

\section{Alternating Structures}

Let $M$ be a differential manifold [4]. Suppose $A$ is a contravariant tensor field of order 2 . In terms of coordinates $x^{1}, \ldots, x^{n}$ for $M$ one can define $\{f, g\}=A^{i j} f_{i} g_{j}$ for any two functions $f, g$ defined on $M$ where $A^{i j}$ are the components of $A, f_{i}$ is $\partial f / \partial x^{i}$ and $g_{j}$ is $\partial g / \partial x^{j}$. Suppose $A$ has the 
properties

$$
\{f, g\}=-\{g, f\}
$$

$$
\{\{f, g\}, h\}+\{\{g, h\}, f\}+\{\{h, f\}, g\}=0 .
$$

Then we call $A$ an alternating structure for $M$ and call $\{f, g\}$ the Poisson bracket of $f$ and $g$ defined by $A$. The implication of 2.1 is obvious. The meaning of 2.2 has been explored in [7].

We call the alternating structure $A$ regular if in some coordinate system (and hence all) the determinant of $A^{i j}$ is never 0 . Of course this implies that $M$ is even-dimensional. The classical Poisson bracket is regular. When $A$ is regular one can define a 2-form $a_{i j} d x^{i} \wedge d x^{j}$ where $\left(a_{i j}\right)$ is the inverse of the matrix $\left(A^{i j}\right)$. For regular alternating structures it follows from 2.2 that $^{1}[7, \S 11]$

$2.3 a_{i j} d x^{i} \wedge d x^{j}$ is closed, which is equivalent to

$$
\frac{\partial a_{i j}}{\partial x^{k}}+\frac{\partial a_{j k}}{\partial x^{i}}+\frac{\partial a_{k i}}{\partial x^{j}}=0 .
$$

The proof of this can be easily supplied by the reader.

A function $h$ is called a generating function for a vector field $Z$ if for every $f$ one has $\{h, f\}=Z f$. In terms of coordinates and components, a necessary and sufficient relation is

$$
Z^{i}=A^{j i} \frac{\partial h}{\partial x^{j}} .
$$

If $A$ is regular, this is tantamount to

$$
d h=Z^{j} a_{j k} d x^{k} .
$$

We say that $Z$ has generating functions locally if, in a neighbourhood of any given point, an $h$ can be found such that 2.4 holds. In the regular case this is tantamount to demanding that $Z^{j} a_{j k} d x^{k}$ be closed, that is

$$
\frac{\partial\left(Z^{j} a_{j k}\right)}{\partial x^{i}}=\frac{\partial\left(Z^{j} a_{j i}\right)}{\partial x^{k}} .
$$

Suppose $T$ is a map of $M$ into itself, where $M$ has some alternating structure. If $\{f \circ T, g \circ T\}=\{f, g\} \circ T$, then we say $T$ preserves this structure. If $T_{t}$ is the local one-parameter group generated by an infinitesimal transformation (i.e. vector field) $Z$ in $M$, then $T_{t}$ preserves $\{$, if and only if

$$
\{Z f, g\}+\{f, Z g\}=Z\{f, g\} .
$$

If this holds, we say $Z$ preserves the structure.

\footnotetext{
1 We conform to the summation convention.
} 
If $Z$ has generating functions locally then 2.2 yields 2.5 immediately. In the regular case, the converse holds also.

2.6 If $A$ is regular and $Z$ preserves $A$ then $Z$ has generating functions locally.

The proof consists in establishing 2.42. If $M$ is simply-connected, a global generating function exists for such $Z$. If $A$ is not regular, not even local generating functions need exist.

Even when the closed 2-form 2.3 is not regular, 2.42 is the condition that $Z$ preserve it. It would be unwarranted, however, to describe the relation 2.41 between $Z$ and $h$ in the non-regular case by saying that $h$ generates $Z$ because $h$ hardly determines $Z$. We will just say that

\section{$2.7 \quad Z$ and $h$ are $\alpha$-related}

supposing that the 2 -form 2.3 involved in 2.41 be called $\alpha$. (When $\alpha$ is regular it is often called a symplectic structure [5].)

\section{Hamiltonian Actions}

A group $G$ is said to act in a space $M$ if there is defined a "product" $T m$ for $T$ in $G$ and $m$ in $M$ with values in $M$ such that $S(T m)=(S T) m$. If $G$ is a Lie group and $M$ is a manifold, suitable differentiability is tacitly required. In any case, one requires enough differentiability such that if $T_{t}$ is a one-parameter subgroup of $G$, then there is a vector field $\Delta^{\prime}$ in $M$ such that

$$
\left.\frac{d}{d t} f\left(T_{t} m\right)\right|_{t=0}=\left(\Delta^{\prime} f\right)(m)
$$

for all real-valued (differentiable) functions $f$ on $M$. (If, for example, $G$ is acting on itself by left multiplication - the left action - then $\Delta^{\prime}$ will be a right invariant vector field on $G$.) If the element $X$ of the Lie algebra of $G$ is the generator of $T_{t}$ we denote $\Delta^{\prime}$ by $\Delta_{X}^{\prime}$.

Now suppose all this holds. Suppose, moreover, $M$ has an alternating structure enabling every such vector field $\Delta_{X}^{\prime}$ to have generating functions locally, then we will call this action a Hamiltonian action. Let $G_{0}$ be the normal subgroup of $G$ containing the elements connected to the identity of $G$. Let $T^{2}\left(M, G_{0}\right)$ be the space of fields of tensors of contravariant degree 2, which are invariant under the action of $G_{0}$ in $M$.

3.2. Proposition. The alternating structures which make the action of $G$ in $M$ a Hamiltonian action are precisely the alternating tensors in $T^{2}\left(M, G_{0}\right)$. 
3.21 Notice. In this paper, we consider only Hamiltonian actions in which the alternating structure is preserved by all of $G$.

A group action is transitive if for each pair of points $m$ and $p$ in $M$ there is at least one $T$ in $G$ such that $T m=p$. In this case $M$ is in $1: 1$ correspondence with the space $G / \Gamma$ of cosets $T \Gamma$ of some subgroup $\Gamma$. In the differentiable situation, transitivity usually ${ }^{2}$ brings with it local transitivity, i.e.,

3.3 At each point $m$ of $M$ the linear space of vectors $\left.\Delta_{X}^{\prime}\right|_{m}$ (where $X$ ranges over the Lie algebra) includes every vector in $M$ at $m$.

3.4. Theorem. Let $G$ act locally transitively in $M$ and suppose that the action is Hamiltonian. Then the alternating structure is regular.

Proof. Let $X_{1}, \ldots, X_{p}$ be a basis for the Lie algebra of $G$. Let $x^{1}, \ldots, x^{n}$ be a system of coordinates in $M$. Let $h_{\alpha}$ be a generating function for $\Delta_{X_{\alpha}}^{\prime}$, in symbols

$$
\Delta_{X_{\alpha}}^{\prime}=\frac{\partial h_{\alpha}}{\partial x^{j}} A^{j i} \frac{\partial}{\partial x^{i}} .
$$

It follows from 3.3 that $p \times n$ matrix of the quantities

$$
\frac{\partial h_{\alpha}}{\partial x^{j}} A^{j i}
$$

must have rank $n$, whence the matrix of the $A$ 's is non-singular, q.e.d.

Now suppose we have an action of $G$ in $M$. An alternating structure $A$ for $M$ which renders this action Hamiltonian may be called a Hamiltonization of it. The following is thus an immediate consequence of 3.4.

3.5. Theorem. Let $G$ act differentiably and locally transitively in $M$. Then the Hamiltonizations of this action are in $1: 1$ correspondence with those invariant (under $G$ ) 2-forms on $M$ which are non-singular.

It may happen that while there are plenty of invariant 2-forms, there are none which are non-singular.

Locally transitive actions in $M$ are easily analysed by noting that $M$ is the disjoint union of open submanifolds on each of which $G$ acts transitively and each of which is in fact equivalent to a coset space $G / \Gamma$.

"Equivalent" here is based on the following. Let $M$ and $N$ be spaces on which $G$ acts and let $U$ map $M$ into $N$. Then $U$ is a $G$-mapping if $U(T(m))=T(U m)$ for every $T$ in $G$ and $m$ in $M$. If $T$ is also onto and $T, T$ are both $G$-mappings then $T$ is an equivalence.

${ }^{2}$ For example, suppose $G$ has at most an enumerable number of components and $M$ is of the second category [4]. 
We want to increase the utility of 3.5 by reformulating it in terms of $G$ alone. The following is a step in that direction.

3.6. Proposition. Let $G$ act differentiably in $M$ and $N$ and suppose $U: M \rightarrow N$ is a differentiable $G$-map. Then in the naturally induced linear mapping of $k$-forms on $N$ into $k$-forms on $M$, closed forms go into closed forms and $G$-invariant forms into $G$-invariant forms.

The induced mapping $U^{*}$ of forms is that in which a form such as $f d g \wedge d h \wedge \ldots$ on $N$ passes into $(f \circ U) d(g \circ U) \wedge d(h \circ U) \wedge \ldots$. Thus this mapping commutes with exterior differentiation ${ }^{3}$. As to the invariance, it is expressed for a given $k$-form (or indeed any covariant vector field) $\alpha$ by a formula involving $k$ "contractions"

$$
\left\langle\left.\alpha\right|_{T m} ; X, Y, \ldots\right\rangle=\left\langle\left.\alpha\right|_{m} ;(d T)^{-1} X,(d T)^{-1} Y, \ldots\right\rangle
$$

where $m$ is a point of $M$ and $X, Y, \ldots$ are $k$ vectors in $M$ at $m$. This formula leads to a short proof of 3.6.

Let us retain the entire hypothesis of 3.6 for a further observation. Suppose $X$ is an element of the Lie algebra of $G$. Then we get a vector field $\Delta_{X}^{\prime}$ in $M$ and also one in $N$. Let us denote these by $X_{M}$ and $X_{N}$. Because $U$ is a $G$-map we will have

$$
\left.X_{N}\right|_{U m}=d U\left(\left.X_{M}\right|_{m}\right) .
$$

But now suppose $X_{N}$ is $\alpha$-related (2.7) to some function $h$ defined on $N$, where $\alpha$ is a 2 -form on $N$. By substitution we obtain a function $h \circ U$ on $M$. The following is easily proved:

3.61. Proposition. $h \circ U$ is $U^{*} \alpha$-related to $X_{M}$.

Let us call the map $U$ locally onto if, for each point $m$ of $M$, every vector at $U(m)$ in $N$ is of the form $d U(X)$ for some vector $X$ at $m$.

The set of those vectors $Z$ in $M$ for which $d U(Z)=0$ shall be denoted by $Z_{U}$, for brevity. Recall that a vector $X$ is called singular for a $k$-form $\alpha$ if $\langle\alpha ; X, Y, Z, \ldots\rangle=0$ no matter what these other $k-1$ vectors are. If a form has no non-zero singular vectors it is regular.

3.7. Theorem. Suppose $U$ is locally onto. Then the map $U^{*}$ establishes $a 1: 1$ correspondence between the closed invariant $k$-forms on $N$ and those closed invariant $k$-forms on $M$ for which each vector in $Z_{U}$ is singular.

Proof. Let $\alpha$ be a non-zero form on $N$. So $\langle\alpha ; X, Y, \ldots\rangle \neq 0$ for some $X, Y, \ldots$. But these are of the form $d U\left(X^{\prime}\right), d U\left(Y^{\prime}\right), \ldots$ and $\left\langle U^{*} \alpha ; X^{\prime}, Y^{\prime}, \ldots\right\rangle$ $=\left\langle\alpha ; d U\left(X^{\prime}\right), \ldots\right\rangle \neq 0$, so $U^{*} \alpha \neq 0$ and $U^{*}$ is shown to be $1: 1$. Next we observe that if $d U\left(X^{\prime}\right)=0$ then $X^{\prime}$ is singular for $U^{*} \alpha$, using the same

${ }^{3}$ This and the other observations on transformation groups are well known. See, for example, [3, § 13]. 
equation. Finally we must show that if $\beta$ has all the vectors in $Z_{U}$ as singular vectors, then it has the form $U^{*} \alpha$. The $\alpha$ can be constructed by defining $\langle\alpha ; X, Y, \ldots\rangle=\left\langle\beta ; X^{\prime}, Y^{\prime}, \ldots\right\rangle$ where $d U\left(X^{\prime}\right)=X, d U\left(Y^{\prime}\right)=Y, \ldots$. The singularity of $\beta$ is enough to make $\left\langle\beta ; X^{\prime}, \ldots\right\rangle$ independent of the choice available for $X^{\prime}, \ldots$.

Having established 3.7, let us now suppose

\subsection{G acts differentiably, transitively and locally transitively in $M$.}

Then the following things will be true (cf. [3]):

3.81 Choose a point $m_{0}$ of $M$. Then the map $G \rightarrow M$ wherein $T$ maps on $T\left(m_{0}\right)$ is locally onto.

3.82 Let $\Gamma$ be the subgroup defined by $T m_{0}=m_{0}$. Then $M$ is G-equivalent to $G / \Gamma$.

3.83 In the resulting $G$-map of $G$ onto $G / \Gamma$, the set $Z_{U}$ (of vectors annihilated) consists of those vectors in $G$ which are tangent to $\Gamma$ or its cosets $T \Gamma$.

The substance of 3.7 can now be stated as follows:

3.9. Theorem. Let 3.8 hold. Find the subgroup $\Gamma$ of 3.81. Then the closed invariant $k$-forms on $M$ are in $1: 1$ linear correspondence with the linear space of those alternating $k$-linear functionals $A$ on the Lie algebra $\mathfrak{g}$ of $G$ for which

3.91 the elements of $\mathrm{g}$ which belong to the Lie algebra of $\Gamma$ are singular and

3.92 if $X_{1}, \ldots, X_{k+1}$ belong to $\mathrm{g}$ then the sum

$$
\sum_{\pi \text { even }} A\left(\left[X_{\pi 0}, X_{\pi 1}\right], X_{\pi 2}, \ldots, X_{\pi k}\right)
$$

extended over all even permutations of $\{0,1,2, \ldots, k\}$ is 0 .

This follows from 3.7 when we observe, first, that 3.92 is true at the identity element of $G$ if and only if the $k$-form is closed; second, any form given at the identity element gives rise to an invariant ${ }^{4} k$-form on $G$; and, third, that 3.91 together with the uniqueness of invariant extension of $A$ to $G$, gives the required singularity of $Z_{U}$.

Now we recall 3.5 and ask which of these alternating 2-forms give rise to Hamiltonizations of $G / \Gamma$. The following is the obvious answer:

\footnotetext{
${ }^{4}$ Here we mean invariant under left translation, of course; for only then is the map $G \rightarrow G / \Gamma$ a $G$-map. 3.9 resembles but differs from [3, Theorem 13.1] which is about invariant forms not necessarily closed. Even so, a formula related to $[3,13.10]$ must hold in our case and is deducible from 3.91 and 3.92. This formula is not explicitly involved in our proof.
} 
3.93. Theorem. Let $A$ be as in 3.9 , where $k=2$. Let $X_{1}, \ldots, X_{n}$ be a basis for $\mathrm{g}$ such that $X_{m+1}, \ldots, X_{n}$ is a basis for the Lie algebra of $\Gamma$. Then $A$ corresponds to a Hamiltonization of $G / \Gamma$ if and only if the determinant of $A\left(X_{i}, X_{j}\right) 1 \leqq i, j \leqq m$, is not 0 .

\section{Examples and Applications}

In order to apply 3.93 , the first thing to do is to get some idea of the closed invariant 2 -forms on $G$. We denote this ${ }^{5}$ linear space by $C^{2}(G)$.

Let $G$ be a Lie group and let $X_{1}, \ldots, X_{n}$ be a basis for its Lie algebra $\mathfrak{g}$, which we suppose is formed by the (left) invariant vector fields on $G$. The Maurer-Cartan $\mu^{i}$ are defined by $\left\langle\mu^{i}, X_{j}\right\rangle=\delta_{j}^{i}$. If $\alpha$ belongs to $C^{2}(G)$ then it can be represented by an antisymmetric matrix $\left(a_{i j}\right)$ such that $\alpha=\sum a_{i j} \mu^{i} \wedge \mu^{j}$. The fact that $\alpha$ is closed forces some linear relations on the $a_{i j}$. Only when these are easy to describe can one conveniently apply 3.93. There are some important groups for which this description is easy. The 2-form $d \mu^{i}$ belongs to $C^{2}(G)$, of course.

Let $\sigma=\left(g_{1}, g_{2}, \ldots, g_{p}\right)$ be a sequence when each $g_{i}$ is \pm 1 . The group of linear homogeneous transformations in $\boldsymbol{R}^{p}$ which preserve $g_{1}\left(x^{1}\right)^{2}$ $+g_{2}\left(x^{2}\right)^{2}+\cdots$ is to be denoted by $O(\sigma)$. The group generated by $O(\sigma)$ together with the translations in $\boldsymbol{R}^{p}$ we denote by $E(\sigma)$ since the Euclidean group is of this form. When $O(\sigma)$ is the Lorentz group then $E(\sigma)$ is the Poincare group. In the case of $O(\sigma)$, a basis $\left\{M_{i j}: 1 \leqq i<j \leqq p\right\}$ for its Lie algebra can be found such that $\left[M_{12}, M_{34}\right]=0,\left[M_{12}, M_{23}\right]=g_{2} M_{13}$ as well as all the other relations obtained by replacing $1,2,3,4$ by any four elements of $\{1,2, \ldots, p\}$. When $M_{i j}$ arises with $i>j$ it means $-M_{j i}$.

In case of $E(\sigma)$ this basis is augmented by $P_{1}, \ldots, P_{p}$ where $\left[P_{i}, P_{j}\right]=0$, $\left[P_{1}, M_{12}\right]=g_{1} P_{2},\left[P_{1}, M_{23}\right]=0$, and so forth $[1$, but delete the $i$ included there for a reason not relevant here].

Speaking more generally now in terms of a basis $X_{1}, \ldots, X_{N}$ of any Lie algebra, we may write down its multiplication table, and in the body of the table replace each $X_{i}$ by a real parameter $A_{i}$. This gives us an $N \times N$ matrix depending on these parameters (when dealing with $E(\sigma)$ it is neater to denote the parameters by $A_{i j}$ and $B_{j}$ ). Any such matrix shall be called compatible with the basis $X_{1}, \ldots, X_{n}$.

4.1. Theorem. Let $X_{1}, \ldots, X_{N}$ be any of the bases described above either for an $O(\sigma)$ or an $E(\sigma)$. Then an $N \times N$ matrix is the matrix of a closed invariant 2-form if and only if it is compatible with the basis.

We prove first that if a 1 be placed in the multiplication table where $X_{i}$ occurs (for some fixed $k$ ) and zeros for all other $X_{j}$, then this is the

${ }^{5}$ A closed invariant 2 -form $\alpha$ is locally of the form $d \beta$, but $\beta$ need not, nor is it required to, be invariant. "Invariant" here, as before, means "left invariant". 
matrix for a closed invariant 2-form $a_{k}$. In fact

$$
a_{k}=2 \Sigma \mu^{i} \wedge \mu^{j}
$$

where this sum is extended over all $i, j$ such that $\left[X_{i}, X_{j}\right]=X_{k}$. Here $\mu^{1}, \ldots, \mu^{N}$ are the Maurer-Cartan forms. It is possible to verify that $a_{k}$ is a multiple of $d \mu^{k}$ (see [2,4]). Since $d\left(d \mu^{k}\right)=0, a_{k}$ is closed. It is surely invariant. It easily follows that every compatible matrix gives a member of $C^{2}(G)$ (and in a 1:1 way since the $a_{k}$ are all different because $X_{k}$ does occur in the table - but this need not concern us at the moment.)

We now turn to the converse. In our table we see, for example, $\left[M_{12}, M_{34}\right]=0$. We must therefore show that

$$
a\left(M_{12}, M_{34}\right)=0
$$

for $a$ in $C^{2}(G)$. Let us abbreviate the left side of 4.11 by $(12,34)$. We ask the reader to write down 3.92 with $\left(X_{0}, X_{1}, X_{2}\right)=\left(M_{14}, M_{24}, M_{34}\right)$ and he will obtain

$$
4.12(34,12)+(23,14)-(13,24)=0 \text {. }
$$

Now he should write down 3.92 using $M_{14}, M_{13}, M_{12}$. This yields

$$
(34,12)+(23,14)+(13,24)=0 \text {. }
$$

The result of these is $(13,24)=0$, which is just as good as 4.11 .

Next consider that $\left[M_{12}, M_{23}\right]=g_{2} M_{13}$ and $\left[M_{14}, M_{34}\right]=-g_{4} M_{13}$. Hence these entries have the relation

$$
g_{4}\left[M_{12}, M_{23}\right]+g_{2}\left[M_{14}, M_{34}\right]=0
$$

whence our assertion requires us to establish

$$
g_{4}(12,23)+g_{2}(14,34)=0 \text {. }
$$

A single appeal to 3.92 suffices, namely using $M_{14}, M_{24}, M_{23}$, as the reader can verify. Of course if $p=3$ then 4.14 does not arise. This finishes the proof of 4.1 for $O(\sigma)$. For $E(\sigma)$ we go on and consider the $P$ 's.

We denote $a\left(P_{i}, M_{j k}\right)$ by $(i, j k)$ and $a\left(P_{i}, P_{j}\right)$ by $(i, j)$. We cannot prove $(1,2)=0$ by using the $P$ 's alone! However, appealing to 3.92 with $M_{12}, P_{2}, P_{3}$ yields $(1,3)=0$.

We observe next that $g_{1}\left[P_{2}, M_{23}\right]=g_{2}\left[P_{1}, M_{13}\right]$. Using 3.92 with $M_{12}, M_{23}, P_{1}$ yields at first $g_{2}(13,1)+g_{1}(2,23)=0$ which gives immediately the desired $g_{1}(2,23)=g_{2}(1,13)$.

Finally, 3.92. Using first $M_{13}, M_{23}, P_{3}$ and then $M_{12}, M_{13}, P_{1}$ gives two equations which combine to show $(2,13)=0$. Thus we have proved that the matrix of components of $a$ is compatible, q.e.d. 
Theorem 4.1 cannot be generalized to all groups. It does not hold for the group of translations. Incidentally, 4.1 says that the groups in question have $E^{2}(G)=0$ in the sense of $[3]^{6}$.

4.2. Corollary. The most general closed invariant 2-form on $O(\sigma)$ is given by$$
\sum A_{k j} g_{i} \mu^{k i} \wedge \mu^{i j}
$$

where $\mu^{i j}$ is the Maurer-Cartan form dual to $M_{i j}$. The sum is extended over all distinct triples in $\{1, \ldots, p\}$. When $\mu^{i j}$ arises with $i>j$, it stands for $-\mu^{j i}$.

The proof consists in observing that an arbitrary linear combination of the $a_{k}$ of 4.1 has the form 4.21 when we switch to the double-index notation appropriate for the basis $\left\{M_{i j}\right\}$.

We can now ask whether the left action of $O(\sigma)$ on itself admits of a Hamiltonization. This is the same as asking whether 4.21 has singular directions.

Our findings are incomplete.

4.3. Theorem. When $N(N-1) / 2$ is odd, every closed invariant 2-form is singular. When $\sigma=(+1,+1, \ldots,+1)$, also. Likewise when $\sigma=(+1,-1,-1,-1)$.

Proof. The degree of the matrix whose determinant determines the singularity is $N(N-1) / 2$. As to $\sigma=(1,1, \ldots, 1)$ we assure the reader that $\sum A_{i j} M_{i j}$ is a singular vector for 4.21 where the $A$ 's here are taken as the very ones in 4.21 . For the case of the Lorentz group, $(1,-1,-1,-1)$, the determinant vanishes identically, by inspection. It does not seem possible to extend this theorem by using the "unitary trick" [3].

We pass to the pseudo-Euclidean groups $E(\sigma)$. The result is again immediate from 4.1 .

4.4. Corollary. The most general closed invariant 2-form for $E(\sigma)$ is given by

$$
\sum A_{k j} g_{i} \mu^{k i} \wedge \mu^{i j}+\sum B_{k} g_{i} \mu^{k i} \wedge \mu^{i}
$$

where in the second sum $k, i$ runs over all distinct pairs and $\mu^{i}$ is dual to $P_{i}$.

4.5. Theorem. On $E(\sigma)$, every closed invariant 2-form is singular.

The proof consists of verifying that $\sum g_{i} B_{i} P_{i}$ is always singular for 4.41. Thus the left action of $E(\sigma)$ on itself does not admit Hamiltonizations.

In order to illustrate the application of 3.93 to cases where $\Gamma$ is not just the identity, we confine ourselves to the case of the Poincaré (or

${ }^{6}$ For the case of $O(\sigma)$, Theorem 4.1 is deducible from [3, Theorem 21.1].

10 Commun math. Phys., Vol. 21 
"inhomogeneous Lorentz") group $E(-1,-1,-1,1)$. As a basis for its Lie algebra we choose

$$
M_{23} M_{31} M_{12} M_{41} M_{42} M_{43} P_{1} P_{2} P_{3} P_{4} .
$$

We now write down the multiplication table, or rather, its body. We will abbreviate $P_{i}$ to $i$ and $M_{i j}$ to $i j$.

4.6. Table

\begin{tabular}{rrrrrrrrrr}
\hline 0 & 12 & -31 & 0 & 43 & -42 & 0 & 3 & -2 & 0 \\
-12 & 0 & 23 & -43 & 0 & 41 & -3 & 0 & 1 & 0 \\
31 & -23 & 0 & 42 & -41 & 0 & 2 & -1 & 0 & 0 \\
\hline 0 & 43 & -42 & 0 & -12 & 31 & -4 & 0 & 0 & -1 \\
-43 & 0 & 41 & 12 & 0 & -23 & 0 & -4 & 0 & -2 \\
42 & -41 & 0 & -31 & 23 & 0 & 0 & 0 & -4 & -3 \\
\hline 0 & 3 & -2 & 4 & 0 & 0 & 0 & 0 & 0 & 0 \\
-3 & 0 & 1 & 0 & 4 & 0 & 0 & 0 & 0 & 0 \\
2 & -1 & 0 & 0 & 0 & 4 & 0 & 0 & 0 & 0 \\
\hline 0 & 0 & 0 & 1 & 2 & 3 & 0 & 0 & 0 & 0
\end{tabular}

According to 4.1 we may regard this array as the matrix of the most general closed invariant 2 -form provided we replace $i$ by some numbers $B_{i}$ and $i j$ by some numbers $A_{i j}$ (and $-i$ by $-B_{i}$ !). Let us now think of this as having been done in 4.6. Let us suppose that $\Gamma$ (see 3.93) is the subgroup generated by $P_{4}$ and $M_{12}$. Then we have to set equal to zero those components in 4.6 which occur in the $P_{4}$ and the $M_{12}$ columns. This is what 3.93 says. So $B_{1}=B_{2}=B_{3}=A_{23}=A_{31}=A_{41}=A_{42}=0$. Then we remove these rows and columns from the matrix, leaving

\begin{tabular}{clcccccc}
\hline 0 & $A_{12}$ & 0 & $A_{43}$ & 0 & 0 & 0 & 0 \\
$-A_{12}$ & 0 & $-A_{43}$ & 0 & 0 & 0 & 0 & 0 \\
\hline 0 & $A_{43}$ & 0 & $-A_{12}$ & 0 & $-A_{4}$ & 0 & 0 \\
$-A_{43}$ & 0 & $A_{12}$ & 0 & 0 & 0 & $-A_{4}$ & 0 \\
0 & 0 & 0 & 0 & 0 & 0 & 0 & $-A_{4}$ \\
\hline 0 & 0 & $A_{4}$ & 0 & 0 & 0 & 0 & 0 \\
0 & 0 & 0 & $A_{4}$ & 0 & 0 & 0 & 0 \\
0 & 0 & 0 & 0 & $A_{4}$ & 0 & 0 & 0 \\
\hline
\end{tabular}

This matrix is regular as long as $A_{4} A_{12} \neq 0$. 
4.7. Theorem. There is a 3-dimensional linear space of closed invariant 2 -forms on $E(-1,-1,-1,1) / \Gamma$ and a 3-dimensional variety of these are regular. There is a 3-dimensional family of Hamiltonizations of the action of $E(-1,-1,-1,1)$ in this coset space.

The subgroup $\Gamma$ here is generated by "time" translations and the rotations in the $x^{1} x^{2}$-plane which preserve orientation. Thus we may say $\Gamma$ is $\boldsymbol{R} \times S O(2)$. The coset space is the union of four copies of $\boldsymbol{R}^{6} \times S^{2}$, $S^{2}$ being the ordinary sphere. 4.7 applies equally well to $\Gamma=E(1) \times S O(2)$, $\boldsymbol{R} \times O(2)$, or $E(1) \times O(2)$. In the last case, the coset space is $\boldsymbol{R}^{6} \times S^{2}$.

Let us now switch $\Gamma$ to be $E(1) \times O(3)$ where this $O(3)$ is presumed working in $x^{1} x^{2} x^{3}$ space. We look at 4.6 and recognize that all $A$ 's and $B$ 's have to be set equal to 0 , except $B_{4}$. The result is as follows:

4.8. Theorem. On the Poincaré group modulo $E(1) \times O(3)$ the space $C^{2}$ is 1-dimensional, and all except the 0 element are regular.

\section{Construction of the Generating Functions}

The possibility of doing so depends on the fact that if $h$ is the generating function on $M=G / \Gamma$ for $\Delta_{X}^{\prime}(X$ in $\mathrm{g})$ then $h \circ \pi$ is a generating function for the vector field ${ }^{7}$ on $G$ corresponding to $X$. More generally, we have the proposition, whose proof is so like that of 3.6 that we omit it.

5.1. Proposition. Let $G, M, N$ and $U$ be as in 3.6. Let $\alpha$ be a closed invariant 2-form on $N$. Let $h$ be $\alpha$-related (2.7) to $\Delta_{X}^{\prime}$ in $N$. Then $h \circ U$ is $\alpha$-related to $\Delta_{X}^{\prime}$ in $M$.

The next theorem tells us what the generating functions are on any Lie group $G$ related to the infinitesimal left multiplications (i.e. rightinvariant vector fields) through the medium of a closed invariant 2-form

$$
\alpha=A_{k} C_{i j}^{k} \mu^{i} \wedge \mu^{j}
$$

where $\mu^{1}, \ldots, \mu^{n}$ are the Maurer-Cartan forms dual to some basis $X_{1}, \ldots, X_{n}$ for $\mathrm{g}$. We let $A$ stand for the column matrix of $A$ 's. Let $\xi$ be a row matrix and consider $X=\xi^{i} X_{i}$.

5.3. Theorem. A function $h$ which is $\alpha$-related to $X$ has the form

$5.31 h=$ const. $+\xi B A$, where $B$ is the transpose of the inverse of the matrix of the adjoint representation of $G$ relative to the basis $X_{1}, \ldots, X_{N}$ for $G$.

By "const." we mean something constant on each connected component of $G$.

${ }^{7}$ The right-invariant vector field $Y$ agreeing with $X$ at $e$ in $G$. 
Proof. For a 2-form $\alpha$ and vector field $Y$ we mean by $\alpha \mid X$ the 1-form $\langle\alpha \mid X ; Y\rangle=\langle\alpha ; X, Y\rangle$. Thus we have (2.41) $d h=\alpha \mid Y$ where $Y$ is $\xi^{p} Y_{p}$ and $Y_{p}$ is the right-translate of $\left.X_{p}\right|_{e}$. We choose canonical co-ordinates $x^{1}, \ldots, x^{n}$ in $G$ in order to use $[2,157]$. It follows from $[2,157]$ that

$$
\begin{aligned}
\mu^{i} & =\left(\frac{e^{\Gamma}-1}{\Gamma}\right)_{j}^{i} d x^{j}, \\
X_{k} & =\left(\frac{\Gamma}{e^{\Gamma}-1}\right)_{k}^{i} \frac{\partial}{\partial x^{i}},
\end{aligned}
$$

and

$$
Y_{k}=\left(\frac{-\Gamma}{e^{-\Gamma}-1}\right)_{k}^{i} \frac{\partial}{\partial x^{i}},
$$

where $\Gamma$ is the matrix such that $\Gamma_{i}^{j}=C_{i k}^{j} x^{k}$.

We observe that $\alpha \mid Y_{p}=A_{k} C_{i m}^{k} \mu^{i}\left(Y_{p}\right) \mu^{m}$. On the right here we use 5.32 and 5.34. After some simplification we obtain

$$
d h=\alpha \mid Y=\left(\xi e^{\Gamma}\right)^{m} A_{k} C_{m i}^{k}\left(\frac{e^{\Gamma}-1}{\Gamma}\right)_{j}^{i} d x^{j} .
$$

We interpolate a lemma.

5.4. Lemma. $d h=\xi d e^{\Gamma}$ A. Here $\Gamma$ etc., are as in 5.3 .

We use a formula in [6] to evaluate $d e^{\Gamma}$. According to [6]

$$
d e^{\Gamma}=e^{\Gamma}\left\{\frac{e^{\Sigma}-1}{\Sigma}\right\} d \Gamma
$$

where $\Sigma$ is $\operatorname{ad} \Gamma$, i.e., $\Sigma(B)=\Gamma B-B \Gamma$ for any $n \times n$ matrix $B$. Now $d \Gamma$ is a matrix; $d \Gamma=C_{k} d x^{k}, \Sigma(d \Gamma)=\Sigma\left(C_{k}\right) d x^{k}$, where $\left(C_{k}\right)_{i}^{j}=C_{i k}^{j}$. Because of the Lie Jacobi relation, $\Sigma\left(C_{k}\right)=\Gamma_{k}^{j} C_{j}$. From this it follows by induction that $\Sigma^{q} C_{k}=\left(\Gamma^{q}\right)_{k}^{j} C_{j}$, where $q$ is any exponent $1,2, \ldots$. This extends to convergent power series, so

$$
\begin{aligned}
& \left\{\frac{e^{\Sigma}-1}{\Sigma}\right\} C_{k}=\left(\frac{e^{\Gamma}-1}{\Gamma}\right)_{k}^{j} C_{j}, \\
& \left\{\frac{e^{\Sigma}-1}{\Sigma}\right\} d \Gamma=\left(\frac{e^{\Gamma}-1}{\Gamma}\right)_{k}^{j} d x^{k} C_{j} .
\end{aligned}
$$

With some change of indices

$$
e^{-\Gamma} d e^{\Gamma}=\left(\frac{e^{\Gamma}-1}{\Gamma}\right)_{j}^{i} d x^{j} C_{i},
$$


whence

$$
\left(e^{-\Gamma} d e^{\Gamma}\right)_{m}^{k}=\left(\frac{e^{\Gamma}-1}{\Gamma}\right)_{j}^{i} C_{m i}^{k} d x^{j} .
$$

This is inserted into 5.34 yielding

$$
d h=\left(\xi e^{\Gamma}\right)^{m} A_{k}\left(e^{-\Gamma} d e^{\Gamma}\right)_{m}^{k}=\left(\xi e^{\Gamma} e^{-\Gamma} d e^{\Gamma}\right)^{k} A_{k},
$$

from which 5.4 follows.

To complete the proof of 5.3 we next show that, on the connected component of the identity in $G$,

$5.5 e^{\Gamma}$ is the transpose of the inverse of the adjoint representation of $G \mathrm{in} \mathfrak{g}$.

To see this we first show that (see [4] for "ad")

$5.51 \Gamma$ evaluated at $\exp X$ in $G$ is the negative of the transpose of the matrix of $\operatorname{ad} X$.

Proof. Relative to the basis $X_{1}, \ldots, X_{N}$, the matrix of an operator $V$ is $M_{j}^{i}$ where $V\left(X_{i}\right)=M_{j}^{i} X_{j}$ (see [6].) Now suppose $X=\xi^{p} X_{p}$. Then ad $X\left(X_{i}\right)=\xi^{p}\left[X_{p}, X_{i}\right]=\xi^{p} C_{p i}^{j} X_{j}=-C_{i p}^{j} \xi^{p} X_{j}$. On the other hand, the value of $\Gamma_{i}^{j}=C_{i p}^{j} x^{p}$ at $\exp \xi^{p} X_{p}$ is $C_{i p}^{j} \xi^{p}$ because the canonical coordinate $x^{k}$ has the value $\xi^{k}$ at $\exp X$. This proves 5.51 .

Now let $\Gamma^{\prime}$ be the negative of the transpose of $\Gamma$. Then $e^{\Gamma^{\prime \prime}}$ evaluated at $T=\exp X$ is the matrix of $e^{\operatorname{ad} X}$. From $[4,118]$ we note that $e^{\text {ad } X}$ $=\operatorname{Ad}(\exp X)=\operatorname{Ad}(T)$, so $e^{\Gamma^{\prime}}$ at $T$ is the matrix of $\operatorname{ad} T$ from which 5.3 follows.

We repeat that this does not give us all the conceivable generating functions when dealing with closed invariant 2-forms not of the type 5.2. But we have seen in Section 4 that the Poincaré group and many others have no other closed invariant 2 -forms than these.

\section{Constants of the Motion}

In the traditional case, when there is a Poisson bracket and a Hamiltonian $h$, a function $f$ defined on the phase space is called $a$ "constant of the motion" if $\{f, h\}=0$. When the entire space-time group is brought in this definition gives rise to another, namely that $f$ "is the same for all observers" and the defining condition is that $\Delta_{X_{i}}^{\prime} f=0$ for all $X_{i}$ in the Lie algebra.

When the action is transitive, this means that $f$ is constant on each component of $M$, or that $d f=0$.

When the action is Hamiltonian and $\Delta_{X_{i}}^{\prime}$ has the generating function $h_{i}$, then such invariant functions are usually discovered by seeing if 
$\left\{f, h_{i}\right\}=0$ for each $i$. When the $h_{i}$ have the same commutation relation as the $X_{i}$, this comes down to

$6.1 \frac{\partial f}{\partial h_{i}} C_{i m}^{k} h_{k}=0$ for all $m$; and supposing that $f$ depends only on $h_{i}, \ldots, h_{n}$.

We wish to point out that even in the irregular case (when there is no Poisson bracket) the condition 6.1 is still sufficient to prove $f$ locally constant, i.e., $d f=0$.

6.2. Theorem. Let $G$ be a Lie group. Let $\alpha$ be as in 5.2. Then the functions $h_{i}$ which are $\alpha$-related to the infinitesimal left translations on $G$ can be so selected that 6.1 implies $d f=0$.

Proof. We take the constant in 5.31 to be 0 , so that $h_{p}=A_{j}\left(e^{\Gamma}\right)_{p}^{j}$. A simple calculation using 5.32-5.34 shows that $\left\langle\mu, Y_{p}\right\rangle=h_{p}$ as well. We now evaluate $Y_{m} h_{k} . Y_{m} h_{k}=\left\langle Y_{m} ; d h_{k}\right\rangle=\left\langle\alpha \mid Y_{k} ; Y_{m}\right\rangle=\left\langle 2 d \mu ; Y_{m}, Y_{k}\right\rangle$ where $\mu=A_{k} \mu^{k}$. This shows $Y_{m} h_{k}=-Y_{k} h_{m}$. It is a general law that $\left\langle 2 d \mu ; Y_{m}, Y_{k}\right\rangle=\left\langle\mu ;\left[Y_{k}, Y_{m}\right]\right\rangle-Y_{k}\left\langle\mu, Y_{m}\right\rangle+Y_{m}\left\langle\mu, Y_{k}\right\rangle$. We observe that $\left[Y_{k}, Y_{m}\right]=-C_{k m}^{i} Y_{i}$. Thus $Y_{m} h_{k}=-C_{k m}^{i} h_{i}+2 Y_{m} h_{k}$. Thus $Y_{m} h_{k}$ is a constant times $C_{m k}^{i} h_{i}$. From this we see that the expression in 6.1 is a constant times $Y_{m} f$. Thus, if 6.1 is assumed then $d f=0$.

Acknowledgements. This research was supported by NSF grant 18127. The author is grateful to Professor Abdus Salam, the International Atomic Energy Agency, and UNESCO, at the International Centre for Theoretical Physics, Trieste, where the report was prepared.

\section{References}

1. Bargmann, V., Wigner, E. P.: Group-theoretical discussion of relativistic wave equations, Proc. Natl. Acad. Sci. U.S. 34, 211-223 (1948).

2. Chevalley, C.: Lie groups, Princeton U.P. 1946.

3. - Eilenberg, S.: Cohomology theory of Lie groups and Lie algebras, Trans. Am. Math. Soc. 63, 85-124 (1948).

4. Helgason, S.: Differential geometry and symmetric spaces. New York: Academic Press 1962.

5. Sternberg, S.: Differential geometry. Englewood Cliffs, N. Jersey: Prentice Hall 1964.

6. Arens, R.: Invariant sublogics as a way from scalar to manycomponent wave equations, J. Math. Mech. 15, 344-372 (1966).

7. - A quantum-dynamical relativistically-invariant rigid body system, Trans. Am. Math. Soc. 147, 153-201 (1970).

R. Arens

Department of Mathematics

University of California

405 Hilgard Avenue

Los Angeles, Cal. 90024 USA 